\title{
ESTUDO EXPLORÁTORIO DA FRAGILIDADE AMBIENTAL DO PARQUE ESTADUAL DA SERRA DO ROLA MOÇA - MG UTILIZANDO ESTATÍSTICA MULTIVARIADA
}

Amanda Alves dos Santos*

\section{Resumo}

Os estudos de fragilidade ambiental devem conter análises representativas do meio ambiente em seu sentido mais amplo considerando os fatores físicos, bióticos e abióticos. Ciente disso, este trabalho apresenta uma proposta metodológica para identificação de áreas de fragilidade ambiental no parque estadual da Serra do Rola Moça com o objetivo de realizar um estudo exploratório indicando, através de métodos de estatística multivariada, áreas específicas que apresentam maior necessidade a proteção. A metodologia empregada foi o método de análise de agrupamento para variáveis categóricas Grade ofMembership (GOM) e os resultados obtidos foram satisfatórios diante do objetivo desse estudo.

Palavras-chave:Fragilidade Ambiental, Estatística Multivariada, Grade ofMembership(GOM)

\begin{abstract}
Studies of environmental fragility must contain analyzes representative of the environment in its broadest sense considering the physical, biotic and abiotic factors. Knowing this, this work presents a methodology for identifying areas of environmental fragility State Park of Serra do RolaMoça with the objective to conduct an exploratory study indicating, through multivariate statistical methods, specific locations that have greater need protection. The methodology used was the method of cluster analysis for categorical variables Grade of Membership (GOM) and the results were satisfactory before the objective of this study.
\end{abstract}

Keywords:Environmental Fragility, Multivariate Statistics, Grade of Membership (GOM)

•Universidade Federal de Minas Gerais - UFMG. Amandalves9@yahoo.com.br 


\section{1- INTRODUÇÃO}

Nos países de economia emergente, a exemplodo Brasil, onde a manutenção dos remanescentes de florestas tropicais passou a ser uma das prioridades governamentais, a criação de áreas sob proteção legal cresceu significativamente. O estabelecimento dessas áreas tem sido uma das mais importantes ferramentas para a conservação de alguns componentes da biodiversidade.

Em âmbito mundial o crescimento do númerode áreas protegidas é o reflexo do aumento de políticas e comprometimento de vários países para conservação da biodiversidade remanescente no planeta terra. O crescimento da rede global de áreas protegidas, comdiversos objetivos de manejo, também reflete o alto valor social e cultural dessas áreas para a sociedade em geral.

As unidades de conservação são consideradas,segundo a Lei 9.985, de 18 de julho de 2000, que instituiu o Sistema Nacional de Unidades de Conservação (SNUC) como:

[...] o espaço territorial e seus recursos ambientais, incluindo as águas jurisdicionais, com características naturais relevantes, legalmente instituídos pelo Poder Público, com objetivos de conservação e limites definidos, sob regime especial de administração, ao qualse aplicam garantias adequadas de proteção (BRASIL, 2000, p.1).

Essas unidades são de extrema importânciapara a conservação da biodiversidade e devem ter um manejo adequado para que sua condição de conservação seja assegurada.

Atualmente sabe-se que apesar da grande importância dessas áreas de conservação, os sistemas públicos encontram dificuldades para administrá-las e manejá-las. É grande a discussão entorno deste assunto surgindo até questionamentos sobre a validade da criação de novas unidades de conservação. Apesar da polêmica sobre esse assunto é incontestável a importância dessas áreas sendo necessário, de acordo com Silva (2009), criar novas opções de gestão dessas áreas.

De acordo com o SNUC as unidades de conservação devem ter plano de manejo, o qual deve definir as formas de uso e gestão dessas áreas. Entretanto, ainda hoje são poucas as unidades que apresentam este tipo de planejamento e quando apresentam, na maioria dos casos, os planos contêm uma série de dados desconexos, o que dificulta a ação da equipe responsável pela gestão da unidade. Refletindo sobre essa questão e pensando sobre a necessidade de criar novas opções para gerir essas áreas é que o presente trabalho utiliza uma série de dados produzidos para um plano de manejo com o objetivo de realizar um 
estudo exploratório indicando, através de métodos de estatística multivariada, áreas específicas dentro da unidade que apresentam maior probabilidade à necessidade de proteção.

O estudo da fragilidade ambiental está relacionado com fatores físicos e antrópicos que fornecem subsídios para a indicação de áreas com necessidade de proteção, ou seja, apontam características indicativas da maior ou menor fragilidade da área em questão, sendo que as áreas com maior fragilidade necessitam de maiores cuidados relativos ao seu uso e proteção. Este tipo de análise possibilita uma reflexão sobre as ações necessárias para o planejamento ambiental de acordo com as especificidades da área.

Diante da complexidade da análise da fragilidade ambiental e da necessidade de utilização de vários fatores para realizá-la, a análise empregando multicritérios vem sendo amplamente utilizada como um método capaz de representar a variabilidade das variáveis que representam esse fenômeno.

A análise de multicritério constitui num sistema baseado na combinação de variáveis ou critérios segundo diferentes métodos, ela deve ser utilizada em situações que a análise de apenas uma variável não representa a realidade do fenômeno estudado. O procedimento para implementá-la é baseado no mapeamento de variáveis e na definição dos graus de pertinência, através de lógicas de análise e integração das variáveis, para a construção do resultado final.

As lógicas de análise e integração empregadas variam de acordo com método de análise definido, sendo que alguns métodos são frequentementeutilizados. A lógica booleana é aplicada para o caso de variáveis que apresentam apenas as opções 0 e 1 ou V e F . Já a perspectiva Bayesiana que é pautada no conceito da probabilidade condicional, ou seja, a probabilidade de ocorrência de um fenômeno é medida uma vez que constatada a ocorrência de outro fenômeno a ele correlacionado. Ela é utilizada para o caso de estudos exploratórios onde o pesquisador tem um conhecimento prévio da realidade a ser estudada (Moura. et al, 2011).

Ainda segundo esses autores a média ponderada apresenta grande adequabilidade para estudos ambientais. Neste método é criado um espaço classificatório ordinal, intervalar ou nominal, desde que para isso as variáveis sejam hierarquizadas segundo algum critério de valor. A ponderação das variáveis é realizada por conhecimento de especialistas KnowlegdeDrivenEvaluation ou por conhecimento prévio de situações análogas Data DrivenEvaluation. Outra lógica empregada frequentemente é a lógica fuzzy. A sua aplicação é baseada na premissa de que diante da complexidade ambiental há um fator de 
incerteza na classificação das variáveis que é expresso pelos graus de pertinência variando de 0 a 1 em escala contínua. De acordo com KOAHGURA (2007) essa lógica permite classificar dados e informações imprecisas e ambíguas através de valores lógicos intermediários.

Diante desses métodos e com a intenção de explorar as variáveis obtidas no plano de manejo do Parque Estadual da Serra do Rola Moça de forma a identificar a fragilidade associada ao parque optou-se por utilizar a lógica fuzzy como metodologia através de uma análise que possibilite o agrupamento ou integração de variáveis categóricas. O método definido para esse objetivo foi o Grade OfMembership (GOM).

\section{2 - CARACTERIZAÇÃO DA ÁREA DE ESTUDO}

O Parque Estadual da Serra do Rola Moça está localizado na interseção dos municípios de Belo Horizonte, Nova Lima, Ibirité e Brumadinho, parte central de Minas Gerais, região do quadrilátero ferrífero, porção sul do complexo da Serra do Espinhaço. Com uma área total de 3.942 ha, o parqueestá na junção sistemas serranos e apresenta minério deferro o que lhe confere um alto potencial hidrogeológico, estabelecendo uma importante área de recarga. Essa característica confere ao parque um grande número de nascentes propiciando uma rede de drenagem que faz parte das bacias do Paraopeba e Rio das Velhas. Essas são áreas de mananciais e abastecem parte da região metropolitana de Belo Horizonte. Na carta-imagem da Figura1 é mostrada a localização espacial da área de estudo, no contexto regional e estadual. 


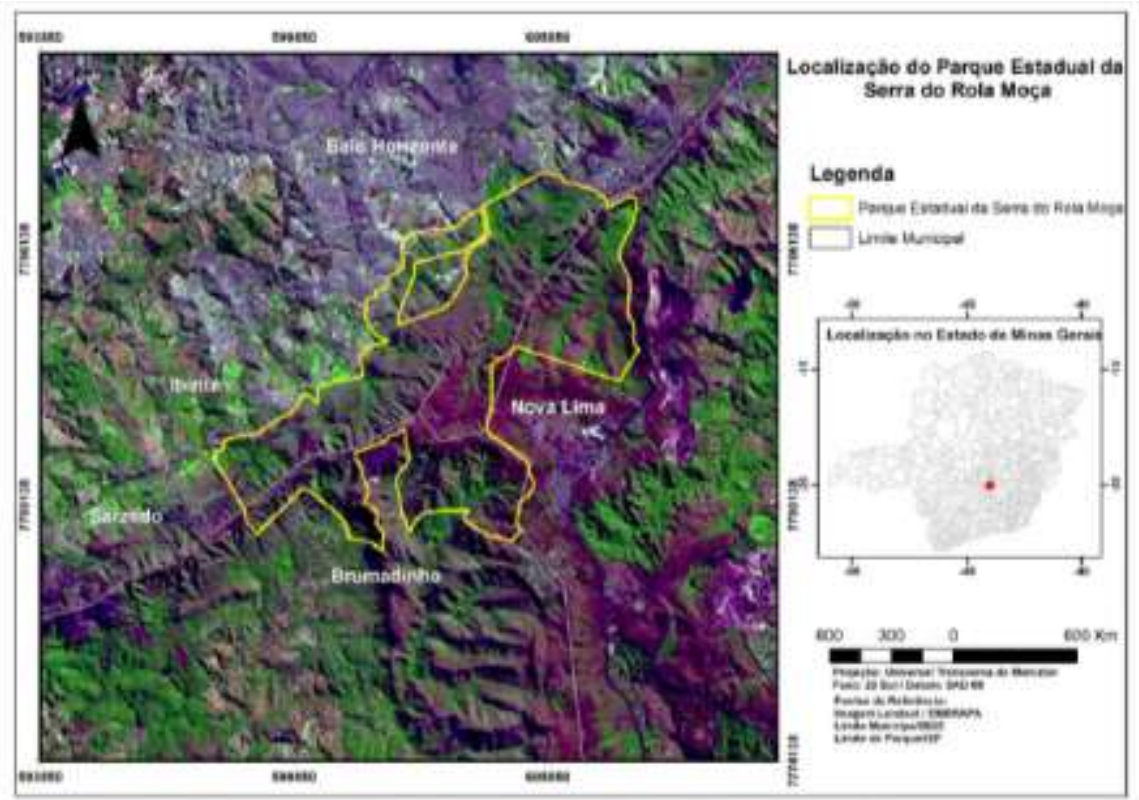

Figura 1 - Mapa de localização do Parque Estadual da Serra do Rola Moça

Além disso, o parque está situado numa área de tensão ecológica entre a Mata Atlântica e o Cerrado, caracterizando uma área importante para conservação da fauna e da flora. De acordo com o Atlas de Áreas Prioritárias para a Conservação da Biodiversidade no Estado de Minas Gerais (DRUMOND, et al, 2005) essa área apresenta importância especial pela presença de ambientes únicos no estado. Do ponto de vista biótico a área apresenta uma série de espécies de fauna e flora ameaçadas e algumas com ocorrência única na área do parque.

Um dos principais problemas do parque está relacionado com o seu entorno. É possível encontrar no entorno próximo, áreas urbanas que muitas vezes são limítrofes com o parque, empresas de mineração, lixões, entre outros. Outro problema recorrente é a incidência de incêndios que são facilmente propagados na área por causa das características vegetacionais, do clima, do relevo e também pela dificuldade de acesso dos bombeiros. Esses são alguns dos problemas recorrentes do parque e que colocam em risco áreas de grande relevância ecológica.

\section{MATERIAIS E METÓDOS}

\subsection{Seleção e descrição das variáveis}

As variáveis foram selecionadas de acordo com os apontamentos do plano de manejo do Parque Estadual da Serra do Rola Moça (DRUMOND, et al,2007) que trazem 
indicações sobre quais das variáveis presentes nesse estudo são capazes de caracterizar a situação ambiental e consequentemente demonstrar o potencial de fragilidade do parque. Neste contexto foram selecionadas 10 variáveis, são elas: proximidade de estradas, proximidade de infraestrutura, pressão antrópica, proximidade de trilhas, proximidade de atrativos, geologia, declividade, proximidade de nascentes, proximidade de recursos hídricos e cobertura vegetal.

As variáveis foram obtidas a partir de planos de informação geográfica elaborados durante o plano de manejo e ou advindos de órgãos públicos de renome. Para obter as variáveis relacionadas à proximidade foi empregado como referência os dados pontuais e lineares dos planos de informação geográfica, como por exemplo: atrativos, estradas e trilhas e utilizado o conceito de áreas de influência no qual são delimitadas faixas entorno da feição geográfica de forma que toda a área de estudo obtenha valores inclusos nessas faixas. Dessa forma, as variáveis continuas puderam ser categorizadas através da definição de intervalos sendo que para cada variável foi definido um intervalo de acordo com suas características, legislação vigente ou pesquisa bibliográfica.

As categorias das variáveis, proximidade de recursos hídricos e proximidade de nascentes foram definidas de acordo com a Lei 14309/2002 que definiu até o ano de 2012 as características das áreas de proteção permanente. Essa legislação foi utilizada porser mais restritiva do que a atual. A Figura 2 representa o mapeamento realizado por áreas de influência através do mapa de proximidade de recursos hídricos. 


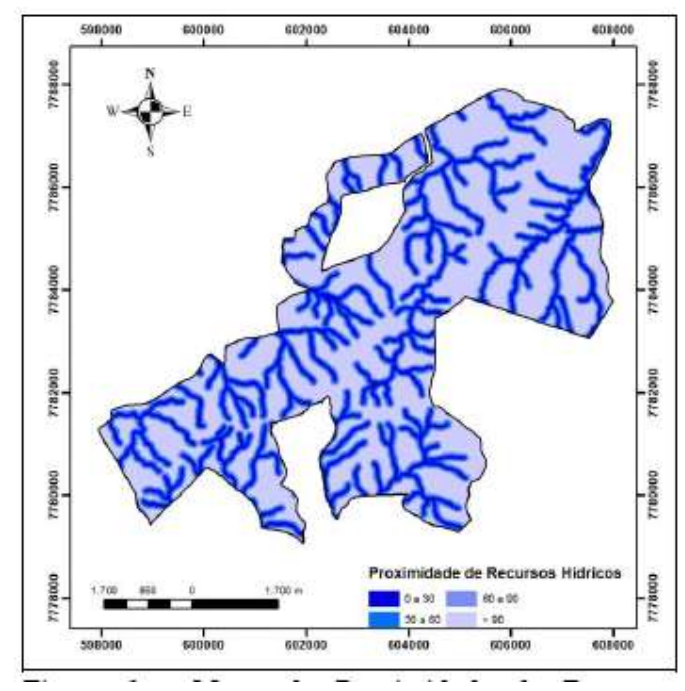

Figura 2 - Mapa de Proximidade de Recursos Hídricos

A variável proximidade de estradas foi categorizada de acordo com o tamanho da área de servidão estipulada pela Lei 6.766/79. Já para proximidade de trilhas, atrativos e infraestrutura foram analisados os textos do plano de manejo em busca de referências que pudessem definir o tamanho da área de influência dessas estruturas. Essas variáveis foram categorizadas em intervalos de até 4 classes como apresentado pela Tabela 1.

Para a variável pressão antrópica foram utilizados pontos classificados pelos pesquisadores do plano de manejo como: alta, média e baixa pressão antrópica e esses valores foram interpolados de forma a obter valores relativos para todo o parque (Figura 3). Jápara declividade foi utilizado um plano de informação de curvas de nível em escala de 1:50.00 e calculado a declividade em graus para a área do parque. Os valores da declividade foram classificados em dois intervalos como sugerido para áreas de APP pela Lei $14309 / 2002$. 


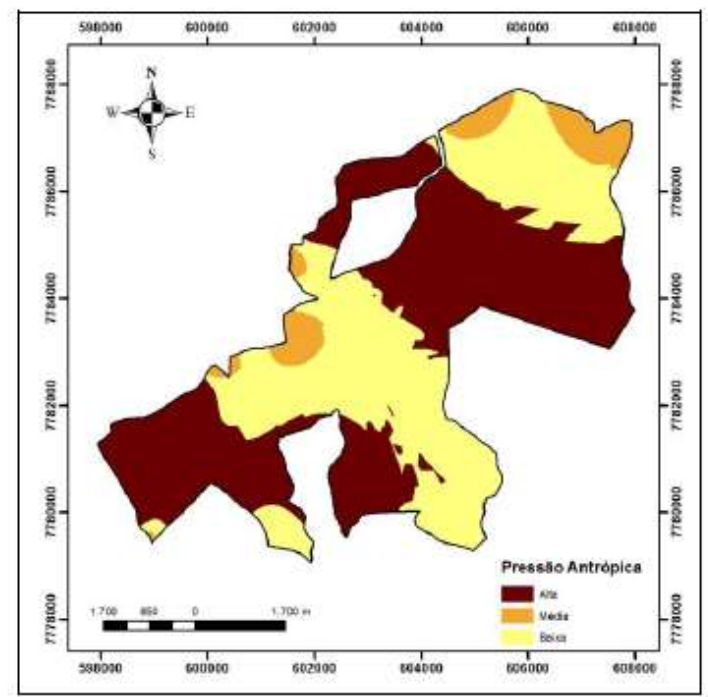

Figura 3 - Mapa de Pressão Antrópica

As variáveis categóricas geologia e cobertura vegetal já estavam espacializadas por toda a área do parque e o único tratamento recebido foi a definição de valores para cada categoria da variável. Esse tratamento também foi realizado para cada categoria ou intervalo das variáveis já mencionadas.

A Tabela 1 a seguir apresenta as variáveis, os intervalos e a numeração representativa de cada categoria. 
TABELA 1 - CATEGORIAS DAS VARIAVEIS DO ESTUDO

\begin{tabular}{|c|c|c|}
\hline Variável & Componentes de Legenda & Categoria \\
\hline \multirow{4}{*}{$\begin{array}{l}\text { Proximidade } \\
\text { de Trillas }\end{array}$} & De 0 a 5 metros & 1 \\
\hline & Des a 10 metros: & 2 \\
\hline & De 10 a 15 metros & 3 \\
\hline & Maior 15 metros & 4 \\
\hline \multirow{4}{*}{$\begin{array}{l}\text { Proximidade } \\
\text { de atrativos }\end{array}$} & De 0 a 100 metros & 1 \\
\hline & De 100 a 200 metros & 2 \\
\hline & De 200 a 300 metros & 3 \\
\hline & Mator que 300 metros & 4 \\
\hline \multirow{4}{*}{$\begin{array}{l}\text { Proximidade } \\
\text { de Estradas }\end{array}$} & De 0 a 15 metros & 1 \\
\hline & De 0 3 30 metros & 2 \\
\hline & De 30 a 45 metros & 3 \\
\hline & Maior que 45 metros & 4 \\
\hline \multirow{4}{*}{$\begin{array}{l}\text { Proximidade } \\
\text { de estradas }\end{array}$} & De 0 a 15 metros & 1 \\
\hline & De 15 a 30 metros & 2 \\
\hline & De 30 a 45 metros & 3 \\
\hline & Acima de 45 metros & 4 \\
\hline \multirow{4}{*}{$\begin{array}{l}\text { Proximidade } \\
\text { de } \\
\text { infraestrunura }\end{array}$} & De 0 a 100 metros & 1 \\
\hline & De 100 a 200 metros & 2 \\
\hline & De 200 a 300 metros & 3 \\
\hline & Maior que 300 metros & 4 \\
\hline \multirow{3}{*}{$\begin{array}{l}\text { Pressào } \\
\text { antrópica }\end{array}$} & Alta & $\mathrm{I}$ \\
\hline & Media & 2 \\
\hline & Baika & 3 \\
\hline \multirow{16}{*}{ Geologia } & Aluvisio: argila & 1 \\
\hline & Canga & 2 \\
\hline & Clonita xisto, clorita-sencita xisto, filito, grauvaca, quartzito & 3 \\
\hline & $\begin{array}{l}\text { Cobertura detrito-lateritica: concreçòes supergènicas de óxidos de } \\
\text { ferro e concreçỏes fernuzinosas preenchendo fraturas }\end{array}$ & 4 \\
\hline & Conglomerado polimitico & 5 \\
\hline & Corpos de minerio de hematita & 6 \\
\hline & Diabásio & 7 \\
\hline & Dolomito, calcino magnesiano; itabinto dolomitico & 8 \\
\hline & Eluvià f fragmentos de itabinto em solo ferruginoso & 9 \\
\hline & Filito & 10 \\
\hline & Gnaisse e granito; migmatito & 11 \\
\hline & Itabinito & 12 \\
\hline & Quartzito & 13 \\
\hline & Sericita-clonita-quartzo xasto & 14 \\
\hline & Talco xisto & 15 \\
\hline & Xisto & 16 \\
\hline \multirow[t]{2}{*}{ Declividade } & $0 \geq 15$ & 1 \\
\hline & Acima de 45 & 2 \\
\hline \multirow{4}{*}{$\begin{array}{l}\text { Proximidade } \\
\text { de Nascente: }\end{array}$} & Até 50 metros: & 1 \\
\hline & $\mathrm{DeSO}=100$ metros & 2 \\
\hline & De 100 a 150 metres & 3 \\
\hline & Maior que 100 metros & 4 \\
\hline \multirow{4}{*}{$\begin{array}{l}\text { Proximidade } \\
\text { de Recursos } \\
\text { Hidnicos }\end{array}$} & Ate 30 metros & 1 \\
\hline & $\mathrm{De} 30$ a 60 metros & 2 \\
\hline & De $60 \geq 90$ metros & 3 \\
\hline & Maior que 90 metros & 4 \\
\hline \multirow{5}{*}{$\begin{array}{l}\text { Cobertura } \\
\text { Vegetal }\end{array}$} & Cenrado & 1 \\
\hline & Floresta Estacional Semi-Decidual & 2 \\
\hline & Savana Gramineo Lenhosa & 3 \\
\hline & Refüzio Ecológico & 4 \\
\hline & Areas Degradadas & 5 \\
\hline
\end{tabular}

\subsection{Descrição da Metodologia}

Como descrito anteriormente os valores relacionados a cada variável estavam associado a planos de informação geográfica em formato vetor. Para obter esses valores as variáveis foram interceptadas de forma a gerar um plano de informação com uma tabela 
onde cada linha representa um registro com dados de todas as variáveis. Foram identificados 5287 registros.

Para a identificação dos agrupamentos e descrição das diferenças entre os mesmo foi utilizado o método de estatística multivariada Grade OfMembership (GOM). Este é um método de modelagem de dados categóricos que permite o agrupamento dos registros baseado na lógica fuzzy. Nos agrupamentos fuzzy cada padrão de dados é associado a um cluster a partir de uma função de pertinência, o que possibilitaque cada registro possa pertencer parcialmente, ou seja,

apresentar graus de pertinência múltiplos em relação aos clusters. Essa forma de agrupar os dados é bastante aplicável para o contexto ambiental, pois apresenta a possibilidade de existência de faixas de transição entre grupos o que se apresenta como uma característica real neste contexto.

Segundo Garcia (2000) o método baseia-se na probabilidade multinominal para estimar dois parâmetros: o grau de pertencimento a um grupo (gik), denominado de perfil neste método e a probabilidade de ocorrência ( $\lambda \mathrm{kj} \mathrm{j})$.

De acordo com Cardoso et.al (2011) cada registro é caracterizado por graus de pertencimento ou escores GOM denominados gik que indicam o grau de pertencimento $(\mathrm{g})$ a cada elemento (i) de cada perfil (k).Esse escores variam num intervalo de 0 a 1 e estão associados aos perfis. Um elemento terá a quantidade de gik igual ao número de perfis definidos. Quanto mais próximo de 1 o valor do gik estiver em relação a um perfil, maior o grau de associação em relação a este e consequentemente menor a associação em relação aos demais. Um gik igual a 1 indica que o elemento está totalmente associado ao perfil específico e igual a 0 indica que não há associação.

Os perfis extremos ou puros são caracterizados pelas probabilidades de ocorrência ( $\lambda$ kjl), ou seja, pela probabilidade ( $\lambda$ ) de ocorrência de cada categoria (l) da variável (j) a cada perfil $(\mathrm{k})$. Essas probabilidades são baseadas nos registros com gik=1 ou seja nos perfis puros e constituem-se em parâmetros para o grupo, além disso elas fornecem a magnitude da associação de cada categoria da variável ao perfil. Este parâmetro também assume valores entre 0 e 1.

Neste estudo os valores de gik e $\lambda$ kjl foram estimados através do software GOM3 que utiliza o algoritmo Woodbury 1974 de forma iterativa para estimar os escores e as probabilidades já descritos. Esse procedimento foi realizado para 3, 4, 5 e 6 perfis.

Para a definição do número de perfis foram aplicados dois métodos, o critério de Akaike no qual o menor valor indica o modelo com melhor ajuste e o cálculo do número 
de perfis puros, ou seja, aqueles com gik $=1$. Esse cálculo identifica a quantidade de perfis com maior número perfis puros e, portanto o melhor ajuste para o modelo.

Os parâmetros do gik estimados foram espacializados de forma a obter uma representação geográfica do perfil em que se encontra o maior valor de gik para cada elemento.

\section{4 -RESULTADOS}

Os resultados do cálculo do número de perfis puros e do critério de Akaike (AIC) foram aplicados de forma complementar e convergiram para utilização de 3 perfis. As tabelas 2 e 3 apresentam, respectivamente, os resultados dos dois métodos utilizados para a definição dos perfis:

TABELA 2 - NÚMERO DE PERFIS PUROS
\begin{tabular}{|r|r|r|r|r|}
\hline \multirow{3}{*}{$\boldsymbol{k}$} & \multicolumn{5}{|c|}{ Número de perfis } \\
\cline { 2 - 5 } & 3 & $\mathbf{4}$ & $\mathbf{5}$ & $\mathbf{6}$ \\
\hline $\mathbf{1}$ & 1008 & 349 & 417 & 357 \\
\hline $\mathbf{2}$ & 1183 & 633 & 302 & 211 \\
\hline 3 & 934 & 413 & 433 & 303 \\
\hline 4 & & 412 & 308 & 304 \\
\hline $\mathbf{5}$ & & & 417 & 322 \\
\hline $\mathbf{6}$ & & & & 249 \\
\hline Total & 3125 & $\mathbf{1 8 0 7}$ & 1877 & 1746 \\
\hline
\end{tabular}

TABELA 3 - CRITÉRIO DE AKAIKE
\begin{tabular}{|r|c|c|c|c|}
\hline \multicolumn{1}{c|}{$k$} & $n$ & $\boldsymbol{p}$ & $\ln (\boldsymbol{L})$ & AIC \\
\hline 3 & 5287 & 15405 & $-49636,1$ & 130082,3 \\
\hline 4 & 5287 & 20538 & $-46271,4$ & 133618,8 \\
\hline 5 & 5287 & 25675 & $-44531,8$ & 140413,7 \\
\hline 6 & 5287 & 30810 & $-43284,3$ & 148188,6 \\
\hline
\end{tabular}

Como é possível observar na Tabela 2 o número de perfis puros para $\mathrm{k}=3$ é consideravelmente maior do que para os outros perfis e representa 59\% dos registros analisados enquanto que, para os outros perfis essa porcentagem varia entorno de $30 \%$. Isso indica que para $\mathrm{k}=3$ o modelo encontrou o melhor ajuste de forma a maximizar o número de perfis puros. Para confirmar essa análise foi realizado o cálculo do critério de Akaike como indicativo do melhor número de perfis (Tabela 2).

A análise das características de cada perfil foi realizada a partir da comparação da frequência marginal, que é a frequência de resposta de cada categoria da variável nos dados iniciais, com a probabilidade de ocorrência $\lambda$ kjl. .Essa análise indicou que para o perfil 1 as categorias que apresentam características de maior fragilidade nas variáveis vegetação, declividade, proximidade de recursos hídricos e proximidade de nascentes tiveram maiores probabilidades de ocorrência e essas foram maiores que as frequências marginais em todos os casos. Assim esse perfil foi caracterizado como de alta fragilidade. 
No perfil 3 as variáveis proximidades de nascentes, proximidade de recursos hídricos e vegetação também foram representativas entretanto, nesse perfil as categorias das variáveis representam baixa fragilidade. Também para esse caso foram as categorias da variáveis tiveram probabilidade de ocorrência significativas e foram maiores que a frequência marginal. Dessa forma esse perfil foi indicado como de baixa fragilidade.

Já para o perfil 2, para quase todas as variáveis os valores de probabilidade de ocorrência se concentraram, em relação a fragilidade, em torno das categorias intermediárias da variáveis. Esse perfil foi considerado como intermediário entre a alta e a baixa fragilidade e foi denominado como de média fragilidade.

É importante destacar que, as variáveis geologia, proximidade de recursos hídricos, proximidades de nascentes e cobertura vegetal se apresentam como indicadoras diferenças entre os grupos. Já as variáveis de característica antrópica ou relacionada ao turismo apresentam probabilidades de ocorrência com valores próximos o que dificulta a discriminação entre os grupos. As variáveis declividade, proximidade de atrativos e proximidade de infraestrutura apresentaram probabilidade de ocorrência $\lambda$ kjl iguais a 1 para dois perfis o que impossibilitou a utilização dessas para a discriminação dos perfis 2 e 3 . Acredita-se que para a variável declividade isso tenha ocorrido devido a generalização a qual agrupou os valores dessa variável no momento da definição do número de classes.

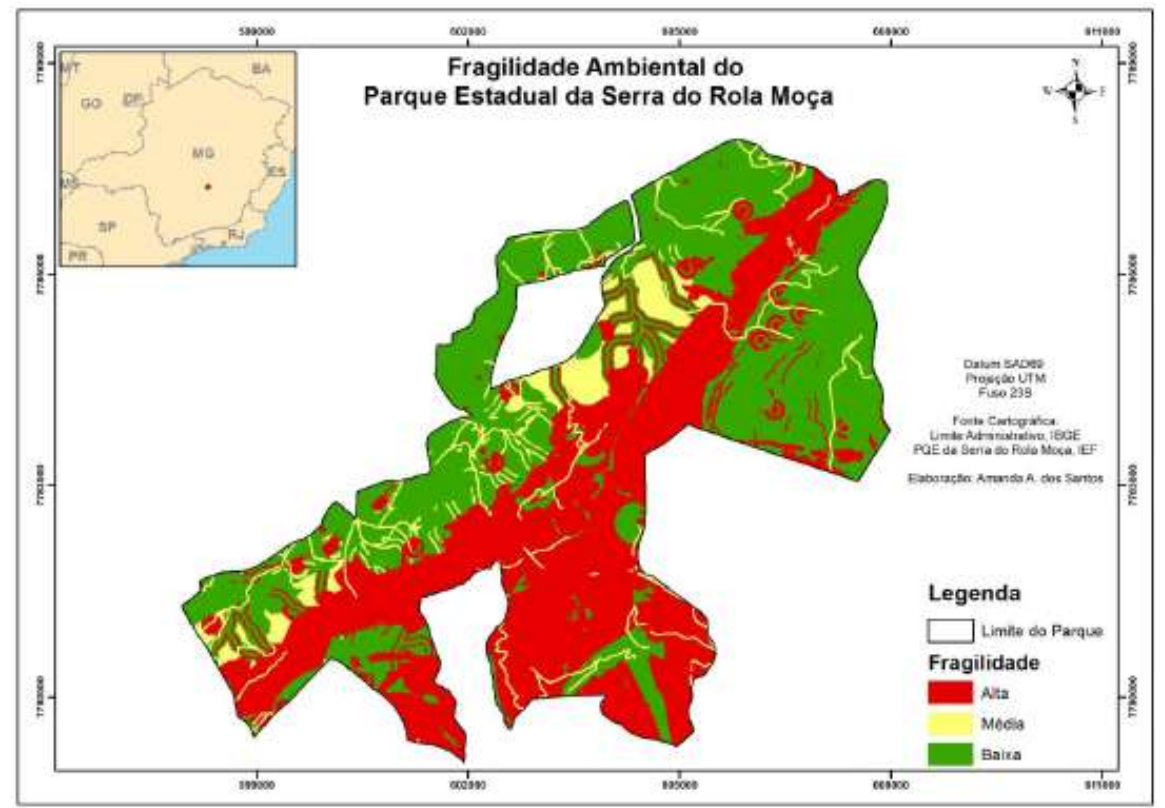

Figura 4 - Mapa de Fragilidade Ambiental 
A Figura 4 apresenta a espacialização dos perfis de fragilidade ambiental do Parque Estadual da Serra do Rola Moça. Através desse mapa é possível observar que o perfil de alta fragilidade está situado, principalmente, vertente sul do parque onde está localizado uma quantidade expressiva de nascentes e cursos d'agua, além disso, essas áreas apresentam altas declividades e uma vegetação muito frágil que é caracterizada principalmente por refúgios ecológicos e savana gramíneo-lenhosa.

As faixas de fragilidade média estão localizadas, principalmente, próximo as faixas intermediárias de recursos hídricos, trilhas e estradas. Já as áreas de baixa fragilidade localizam-se principalmente na vertente norte do parque e estão relacionadas com uma vegetação mais resistente e áreas menores declividades.

\section{5- CONSIDERAÇÕES FINAIS}

O método de análise empregado apresenta sua relevância pautada no fato de realizar uma análise com multicritérios tendo por princípio as características inerentes do conjunto de dados e tendo uma menor interferência do pesquisador, entretanto, considerando que essa análise utiliza como base de dados planos de informações geográficas a utilização do software GOM3 apresenta algumas restrições.

Em análises espaciais que utilizam multicritérios é normalmente empregado a discretização dos planos de informação de forma a obter informações para cada pixel, sendo que este pode variar de tamanho dependo da resolução a base cartográfica. Neste estudo optou-se por utilizar a interseção de vetores devido a grande quantidade de registros gerados a partir da transformação de dados raster em tabelas e a dificuldade do software de trabalhar com grandes conjuntos de dados. Este fato pode ter tido influência na geração de faixas quase que continuas de fragilidade.

Outro aspecto importante é a quantidade de variáveis. Segundo Caetano e Machado (2009) para melhor estimar as probabilidades de ocorrência $\lambda$ kjl é necessário ter maiores números de observações enquanto que para melhor estimar os graus de pertencimento gik precisa-se de maior número de variáveis, ainda segundo esses autores a combinação dos dois apresenta o melhor ajuste para o GOM. Como a analise aqui apresentada utilizou apenas 10 variáveis este pode não ter sido o melhor ajuste para o modelo de fragilidade ambiental do Parque Estadual da Serra do Rola Moça.

Apesar dos itens pontuados acima, essa análise pode ser considerada como um importante estudo exploratório sobre as informações ambientais disponíveis do Parque Estadual da Serra do Rola Moçae como sugestão de uma forma de análise que realize o 
agrupamento dos dados, já que esses são normalmente analisados de forma separada dificultando a visão integrada das características do parque. Além disso, é apresentada uma possibilidade de aplicação, para dados ambientais, de um método de estatística multivariada específico para variáveis categóricas o GOM.

\section{REFERÊNCIAS BIBLIOGRÁFICAS}

BRASIL. Lei n 6.766 , de 19 de dezembro de 1979. Dispõe sobre o parcelamento do solo e dá outras providências

BRASIL. Lei n 14.309, de 19 de junho de 2002. Dispõe sobre as políticas florestais e de proteção à biodiversidade no Estado.

BRASIL. Lei n 9.985, de 18 de julho de 2000. Regulamenta o art. 225, \1o, incisos I, II, III e VII da Constituição Federal, institui o Sistema Nacional de Unidades de Conservação da Natureza e dá outras providências.

CAETANO, A. J; MACHADO, C. J. Consistência e identificabilidade no modelo Grade ofMembership: uma nota metodológica. Revista Brasileira de Estudos Poulacionais. Rio de Janeiro,v.26, n.1, p.145-149, jan/jun.2009.

CARDOSO, L.O. et al. Uso do método Grade ofMembership na identificação de perfis de consumo e comportamento alimentar de adolescentes do Rio de Janeiro, Brasil. Cad. Saúde Pública, Rio de Janeiro, v. 27, n. 2, Feb. 2011.

DRUMMOND, G. M. et al. Fundação Biodiversitas. Biodiversidade em Minas Gerais. 2. ed. Belo Horizonte: Fundação Biodiversitas, 2005. 222p.

DRUMOND, G.M. et al. Plano de Manejo do Parque Estadual da Serra do Rola Moça, Incluindo a Estação Ecológica de Fechos. 1.ed. Belo Horizonte. Fundação Biodiversitas, 2007.

GARCIA, R.A. Modernização e crescimento populacional nos municípios de Minas Gerais: uma aplicação do método "Grade ofMembership". Diamantina: Cedeplar/Ufmg 2000.

GUEDES, R.G. et al. Identificabilidade e estabilidade dos parâmetros no método Grade ofMembership (GOM: considerações metodológivas e práticas. Revista Brasileira de Estudos Poulacionais. Rio de Janeiro,v.27, n.1, p.21-33, jan/jun.2010.

MOURA, A.C.M.; Santa Cecília, B.; Pontes, M.Geoprocessamento na requalificação urbana: evolução e contexto no pensamento urbano e estudo de caso no Hipercentro de Belo Horizonte-MG, Brasil. XIII CONFIBSIG - Conferência Iberoamericana de Sistemas de Información Geográfica. Toluca, México. 25 a 28 de maio, 2011. 16 p.

KOHAGURA, T. Lógica fuzzy e suas aplicações. 2007. Monografia (Graduação em Ciência da Computação) - Universidade Estadual de Londrina; Londrina. 